\title{
Transient Climate Response in Coupled Atmospheric-Ocean General Circulation Models
}

\author{
MAO-CHANG LIANG \\ Research Center for Environmental Changes, and Institute of Astronomy and Astrophysics, Academia Sinica, \\ Taipei, and Graduate Institute of Astronomy, National Central University, Jhongli, Taiwan \\ LI-CHING LIN \\ Institute of Earth Sciences, Academia Sinica, Taipei, Taiwan \\ KA-KIT TUNG \\ Department of Applied Mathematics, University of Washington, Seattle, Washington \\ YUK L. YUNG \\ Division of Geological and Planetary Sciences, California Institute of Technology, Pasadena, California \\ SHAN SUN \\ National Oceanic and Atmospheric Administration/Earth System Research Laboratory, Boulder, Colorado
}

(Manuscript received 11 December 2012, in final form 10 January 2013)

\begin{abstract}
The equilibrium climate sensitivity (ECS) has a large uncertainty range among models participating in the Intergovernmental Panel on Climate Change (IPCC) Fourth Assessment Report (AR4) and has recently been presented as "inherently unpredictable." One way to circumvent this problem is to consider the transient climate response (TCR). However, the TCR among AR4 models also differs by more than a factor of 2 . The authors argue that the situation may not necessarily be so pessimistic, because much of the intermodel difference may be due to the fact that the models were run with their oceans at various stages of flux adjustment with their atmosphere. This is shown by comparing multimillennium-long runs of the Goddard Institute for Space Studies model, version E, coupled with the Hybrid Coordinate Ocean Model (GISS-EH) and the Community Climate System Model, version 4 (CCSM4) with what were reported to AR4. The long model runs here reveal the range of variability $(\sim 30 \%)$ in their TCR within the same model with the same ECS. The commonly adopted remedy of subtracting the "climate drift" is ineffective and adds to the variability. The culprit is the natural variability of the control runs, which exists even at quasi equilibration. Fortunately, for simulations with multidecadal time horizon, robust solutions can be obtained by branching off thousand-year-long control runs that reach "quasi equilibration" using a new protocol, which takes advantage of the fact that forced solutions to radiative forcing forget their initial condition after 30-40 yr and instead depend mostly on the trajectory of the radiative forcing.
\end{abstract}

\section{Introduction}

Very significant research efforts have in the past been devoted to narrowing the uncertainty in the equilibrium climate sensitivity (ECS). While such research has helped

Corresponding author address: Mao-Chang Liang, Nankang P.O. Box 1-48, Taipei 11529, Taiwan.

E-mail: mcl@rcec.sinica.edu.tw our understanding of the feedback processes that contribute to the climate feedback factor $f$, ECS is now known to be "inherently unpredictable" (Roe and Baker 2007) because a small uncertainty in $f$ will lead to a large uncertainty in the high end of the climate gain factor $g=1 /(1-f)$ at equilibrium. This is the reason why ECS has remained stubbornly in the range $1.5^{\circ}-$ $4.5^{\circ} \mathrm{C}$ (Bony et al. 2006; Held and Soden 2000; Solomon et al. 2007) for three decades, with a factor of almost 3 
difference among various Intergovernmental Panel on Climate Change (IPCC) models, and the model uncertainty could actually be even higher (Huybers 2010). Fortunately, with respect to predictions of future warming within a century-which is far from equilibrium - it is the transient climate sensitivity that is more relevant. It has been shown that transient climate response is dependent on ocean inertia-more so than on the fast feedback processes that determine $f$, such as water vapor and cloud feedbacks (Hansen et al. 1985; Held and Soden 2000).

At equilibrium there is in theory no net heat exchange between the ocean and the atmosphere, and a simple mixed-layer ocean (with no ocean dynamics) is commonly used to expedite reaching equilibrium in calculating ECS. Climate predictions of effects of increased emission of greenhouse gases under time-dependent scenarios are affected greatly by the ocean heat uptake. When an ocean module developed with prescribed atmosphere at its interface is coupled to an interacting atmosphere, heat flux exchanges invariably occur at the sea surface.

In very long control runs without forcing trends, there are three stages of evolving climate variability. The first two stages are commonly known as "climate drift," consisting of the "coupling shock"- the large but rapid change when the atmosphere and ocean modules are first coupled, and "millennium drift" - the slow drift that takes several hundred years. These have been discussed elsewhere (Gupta et al. 2012). No models participating in the Fourth Assessment Report (AR4) were run long enough to pass beyond the millennium drift stage. Remedies adopted in AR4 consist mainly of performing a parallel control run with preindustrial conditions alongside a twentieth-century simulation or scenario run and subtracting the temperature trend (the "drift") in the control run from the predicted simulation or scenario run. There is additionally a third stage in very long control runs, "the quasi equilibration," which, despite its name, contains large low-frequency variability.

We performed long integrations using the Goddard Institute for Space Studies model, version E with Hybrid Coordinate Ocean Model [GISS-EHYCOM (GISSEH)] atmosphere-ocean coupled general circulation model (AOGCM) (Shindell et al. 2006; Sun and Bleck 2006) following AR4 protocol; the model is a slightly updated version that GISS-EH used in IPCC AR4 (to correct a wrong implementation of stratospheric ozone trend). We also ran the Community Climate System Model, version 4 (CCSM4), which is well documented (Gent et al. 2012) at T31 resolution for thousands of years. CCSM4 is the model being used for the forthcoming Assessment Report; CCSM3 was used for AR4. In the preindustrial control run, the amount of greenhouse gases and atmospheric aerosol loading is kept at a constant preindustrial level.

\section{Control runs}

Long-term integrations of the control run are shown in Fig. 1, starting with 1850 conditions. For GISS-EH, there was no spinup done prior to the control run (Gupta et al. 2012; Sun and Bleck 2006). The large $0.8^{\circ} \mathrm{C}$ drop during first $20 \mathrm{yr}$ in Fig. 1 (top panel) is followed by a significant secular cooling of $0.7^{\circ} \mathrm{C}$ for the next $350 \mathrm{yr}$ (Shindell et al. 2006). Simulation runs were done for AR4 branching off the control run $200 \mathrm{yr}$ after start (indicated by the red arrow in Fig. 1) in the midst of this negative drift. The drift continues for another $150 \mathrm{yr}$. The model then takes $800 \mathrm{yr}$ to warm and is considered to be "quasi equilibrated" $1200 \mathrm{yr}$ after start. CCSM3 was the model used for AR4 and it has a serious negative drift that persists well into the period of twentiethcentury simulation and future scenario predictions [not shown, but see Gupta et al. (2012)], caused by the incompatibility of modern ocean measurements used to initialize the ocean module with 1870 atmospheric forcing (Gent et al. 2012). This problem has been remedied in CCSM4 (Gent et al. 2012), using $1850 \mathrm{CO}_{2}$ and initial conditions. Figure 1 (bottom panel) shows that CCSM4-T31 has an initial rapid drop of $0.7^{\circ} \mathrm{C}$ in the first $50 \mathrm{yr}$-shorter than that in CCSM3 (Danabasoglu and Gent 2009). It then follows a slow warming of $0.7^{\circ} \mathrm{C}$ for the next $600 \mathrm{yr}$, after which it reaches "quasi equilibration," at which point the 100 -yr trends vary by less than $0.05^{\circ} \mathrm{C}$. CCSM4 has statistically significant long-term ocean variability at 10,100,150, and 400 yr no matter how long the model is run. Even at quasi equilibration CCSM4 has large decadal variability of $0.4^{\circ} \mathrm{C}$ in the control run.

\section{Transient climate response}

Transient climate response (TCR) is defined as the increase in global-mean surface air temperature owing to an imposed $1 \% \mathrm{yr}^{-1}$ increase in $\mathrm{CO}_{2}$ at the time of its doubling. Contributing to the spread in the reported TCR values [phase 3 of the Coupled Model Intercomparison Project (CMIP3); Meehl et al. 2007] is the ocean heat uptake in models at various degrees of ocean adjustment with their atmosphere. The underlying temperature variation from each model's control run has been subtracted, and the last $20 \mathrm{yr}$ averaged, centered at year 70, under the AR4 protocol. Despite the subtraction, the TCR value obtained varies if the control is run longer before applying the $1 \% \mathrm{yr}^{-1}$ increase in $\mathrm{CO}_{2}$ in the TCR experiment. GISS-EH reported a TCR value of $1.6^{\circ} \mathrm{C}$ branching off $200 \mathrm{yr}$ after 

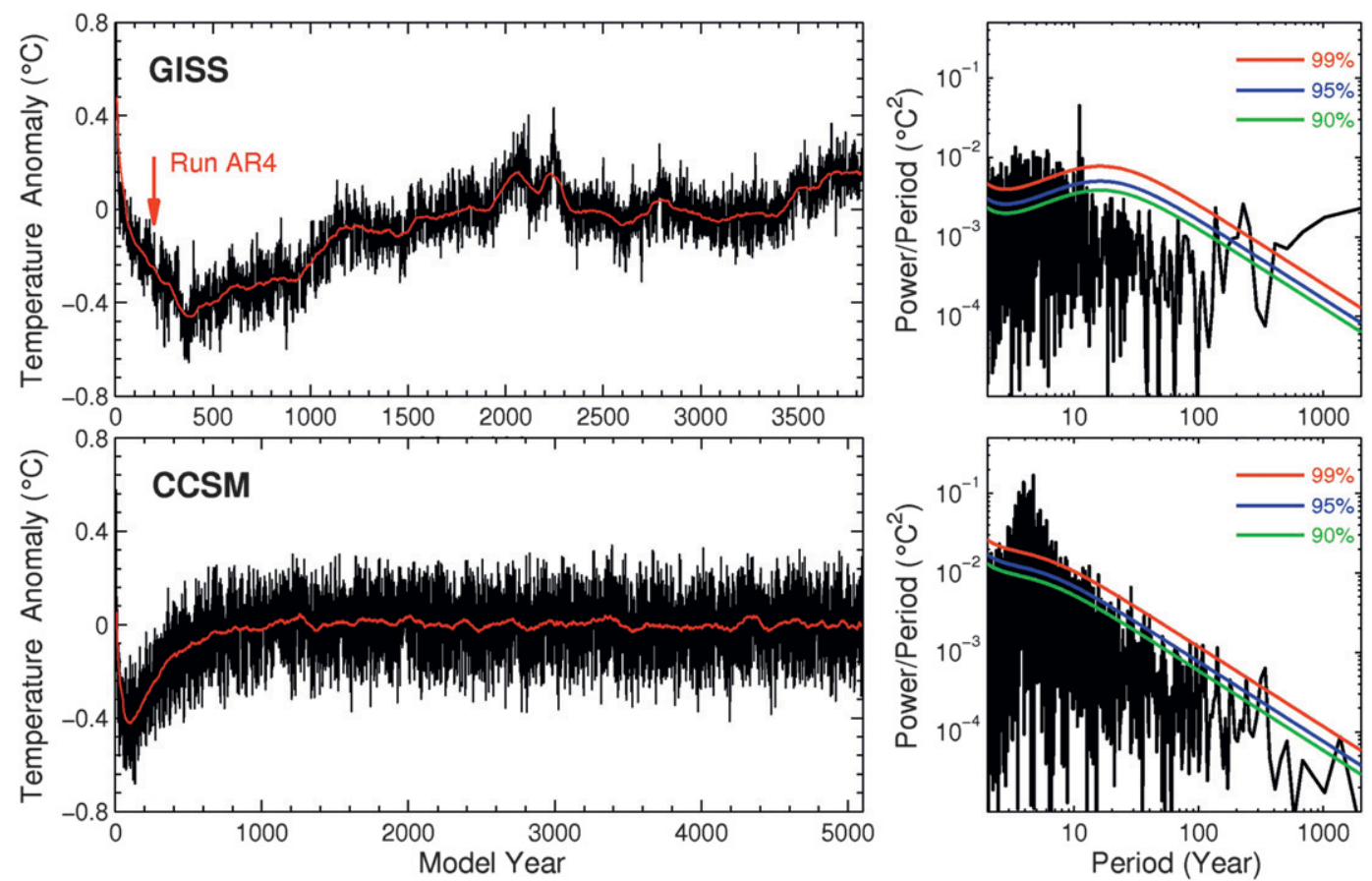

FIG. 1. (left) Global-mean surface air temperature for (top) GISS and (bottom) CCSM4 T31, from multimillennium preindustrial control experiments with no changes in anthropogenic forcing, and (right) its Fourier spectrum (only quasi-equilibrium states are considered). Confidence levels at $99 \%, 95 \%$, and $90 \%$ are shown by the red, blue, and green curves, respectively. The 100-yr running mean is shown in red in the left panels.

the start of the control run, when the climate drift is temporarily low. Figure 2 shows that its TCR values increase by as much as $0.44^{\circ} \mathrm{C}$ when the $1 \% \mathrm{yr}^{-1}$ increase in forcing is applied to the more "equilibrated" states in the control run. The runs done from the equilibrated states still have a variable TCR value (owing to the long-period oscillation still present) but, at about $1.6^{\circ}-$ $2.0^{\circ} \mathrm{C}$, is higher than the one reported to AR4. CCSM3 reported a TCR value for $\mathrm{AR} 4$ of $1.5^{\circ} \mathrm{C}$, while CCSM4 is more sensitive at $1.7^{\circ} \mathrm{C}$ (Bitz et al. 2012; Meehl et al. 2012). Because of its larger internal ocean variability, even at "quasi equilibrium," a larger variation of TCR, $1.5^{\circ}-1.9^{\circ} \mathrm{C}$, is obtained here for the CCSM4. We suspect that there is likely a similar variation of TCR values for individual CMIP3 models. These results then suggest that the single value of TCR that each model reported to AR4 should not be taken as characterizing that model's transient climate sensitivity, since it could be viewed as a random selection from a range of possible values.

Values of TCR for the AR4 participating models range from $1.2^{\circ}$ to $2.6^{\circ} \mathrm{C}$ (Meehl et al. 2007). There are, however, two models with unusually large secular trends in the 100-yr control runs reported to CMIP3 (Meehl et al. 2007). Flexible Global Ocean-Atmosphere-Land System Model gridpoint, version 1.0 (FGOALS-g1.0), which reported a TCR of $1.2^{\circ} \mathrm{C}$, has a total of only $100 \mathrm{yr}$ of coupled spinup (Gupta et al. 2012), during which it shows a problematically large monotonic cooling trend of $-0.54^{\circ} \mathrm{C}$, while the Model for Interdisciplinary Research on Climate 3.2, high-resolution version [MIROC3.2 (hires)], with a reported TCR of $2.6^{\circ} \mathrm{C}$, has a total of only $109 \mathrm{yr}$ of coupled spinup, and an almost monotonic warming trend of $0.14^{\circ} \mathrm{C}$. These are much larger than GISS-EH's linear trend of $0.01^{\circ} \mathrm{C}(100 \mathrm{yr})^{-1}$ or CCMS3's $-0.08^{\circ} \mathrm{C}(100 \mathrm{yr})^{-1}$. Removing the two outliers because of their likely problematic spinup, the range of TCR reported to AR4 is then $1.4^{\circ}-2.2^{\circ} \mathrm{C}$. The range of TCR variability from a single model, $1.5^{\circ}-2.0^{\circ} \mathrm{C}$ for GISS-EH, already spans $63 \%$ of this intermodel spread. Since a single value was reported for each model and it could be from different stages of the spinup, we therefore suspect that the ocean heat uptake variability we have highlighted here is an important factor in understanding intermodel TCR differences. This problem appears potentially solvable, unlike the case of ECS. CCSM3 and GISS-EH have the same ECS of $2.7^{\circ} \mathrm{C}$ (Randall et al. 2007), while CCSM4's ECS is $3.2^{\circ} \mathrm{C}$ (Bitz et al. 2012), and yet their range of TCR is almost the same. This shows that a model's ECS is not a determining factor of its TCR value.

It turns out that a major portion of the variability of TCR values is a result of the way TCR is defined by AR4 and the AR4 protocol of subtracting the underlying 

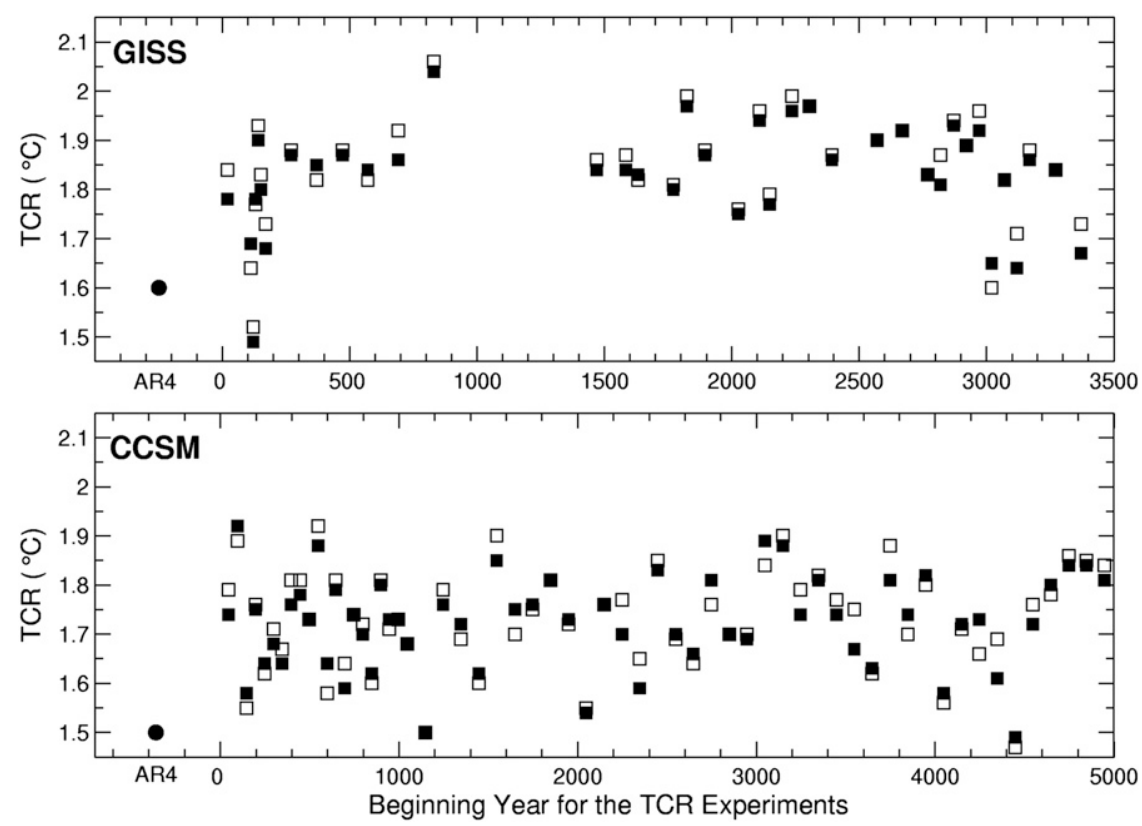

FIG. 2. Comparison of the TCR calculated under AR4 protocol from various years of the GISS-EH and CCSM4 T31 preindustrial control runs, and IPCC AR4 reported values (circles); the CCSM AR4 reported TCR is obtained from CCSM3. The AR4 protocol involves averaging the last $20 \mathrm{yr}$ (solid squares) centered at year 70, and differencing with the year-0 initial condition. Then the underlying temperature of the control run is subtracted. Open squares denote averaging of the last $10 \mathrm{yr}$.

control run. Two subtractions are involved: the subtraction of the year- 0 temperature from the year-70 temperature, followed by the subtraction of the controlrun temperature (year by year during the 70 -yr period). Both subtracted quantities are from the unforced control run, and so the large variability of the control run shown previously in Fig. 1 is transferred to the TCR values thus calculated. The problem is exacerbated by not requiring ensemble averaging in the AR4 protocol for TCR. However, it can be shown that even with ensemble averaging the problem remains unless the AR4 protocol is changed (Liang et al. 2012, manuscript submitted to J. Atmos. Sci.). There it is also shown that the present problem is not due to the chaos or sensitivity to computer configurations (which could have been remedied with ensemble averaging) but to the artifact of climate drift.

In the unforced control run, there is a natural variability in the ocean heat uptake and in surface air temperature. The two are negatively correlated at $r=-0.7$ [Fig. 3, left panel; see also Brierley et al. (2009)]: as more heat goes into the ocean, the surface air temperature decreases. The situation changes when radiative forcing is applied: in the TCR experiment, increasingly higher radiative forcing drives an increasingly larger ocean heat uptake at the same time that the surface temperature warms. Surprisingly, as we have found, radiative forcing of moderate to strong magnitude does not increase the variability beyond the unforced variability, and in many cases even reduces it, as long as the calculation is done past the climate drift stages.

This observation helps us understand the variability of TCR values. For example, two CCSM4 TCRs, one branched off in year 2047 and the other in 3047, and run $80 \mathrm{yr}$ following AR4 protocol of subtracting the respective control-run temperature, differ by a rather large $0.35^{\circ} \mathrm{C}$. However, the cumulative heat fluxes into the ocean integrated over the $70 \mathrm{yr}$ differ by $0.4 \mathrm{~W} \mathrm{~m}^{-2} \mathrm{yr}^{-1}$, which is much smaller than that of the natural variability of the control runs: the control runs during the same 70 -yr periods have a cumulative heat flux difference of $2.6 \mathrm{~W} \mathrm{~m}^{-2} \mathrm{yr}^{-1}$. [The use of the cumulative heat flux is intended only as a simple smoothed measure of average heat flux. For the atmosphere, which has a small thermal inertia, it is the contemporaneous heat flux that is responsible for driving the surface temperature changes (see later).] That increasing $\mathrm{CO}_{2}$ levels can cause a reduction in ensemble range was known (Brierley et al. 2009). Our argument here is that the small heat flux variability in the forced run is not related to the natural variability of the control run, but that latter variability is reintroduced if one subtracts the control-run temperatures from the forced run. 

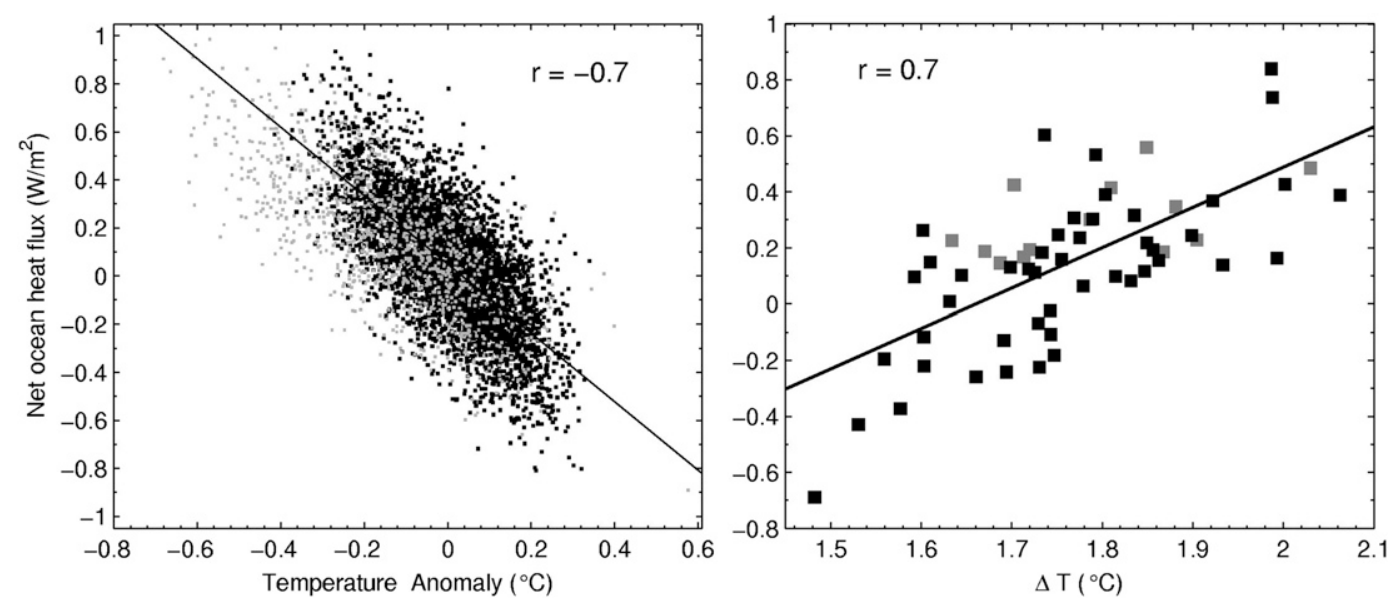

FIG. 3. (left) Scatterplot of annual-mean net ocean heat flux and the corresponding surface air temperature anomaly. (right) Scatterplot of net ocean heat flux at year 0 and $\Delta T=\bar{T}_{70}-T_{0}$. Gray symbols represent values in the spinning-up stage (i.e., first $700 \mathrm{yr}$ of the preindustrial control run).

We shall now use $\Delta T=\bar{T}_{70}-T_{0}$ to denote TCR without the removal of the control run. The term $T_{0}$ is the initial temperature (at "year 0," before the application of the forcing increase). Then the difference in the two TCR values becomes $0.18^{\circ} \mathrm{C}$, which can now be mostly explained by their difference in $T_{0}$. For the present example that difference is from the unforced variability of the control runs at year 2047 and year 3047 in Fig. 1, with the higher TCR value corresponding to the colder $T_{0}$. For all the $\Delta T$ values calculated in the TCR experiments, there is a positive correlation $(r=0.7)$ between $\Delta T$ and the heat uptake at year 0 (Fig. 3, right panel), which is the same correlation as between year0 heat uptake and $-T_{0}$ (left panel), showing that it is the unforced year- 0 heat uptake that controls the TCR variability. Brierley et al. (2009), using a 580-yr run of the third climate configuration of the Met Office Unified Model (HadCM3), also found that the global warming simulated in TCR experiments is correlated with the initial temperature state, and issued a warning that
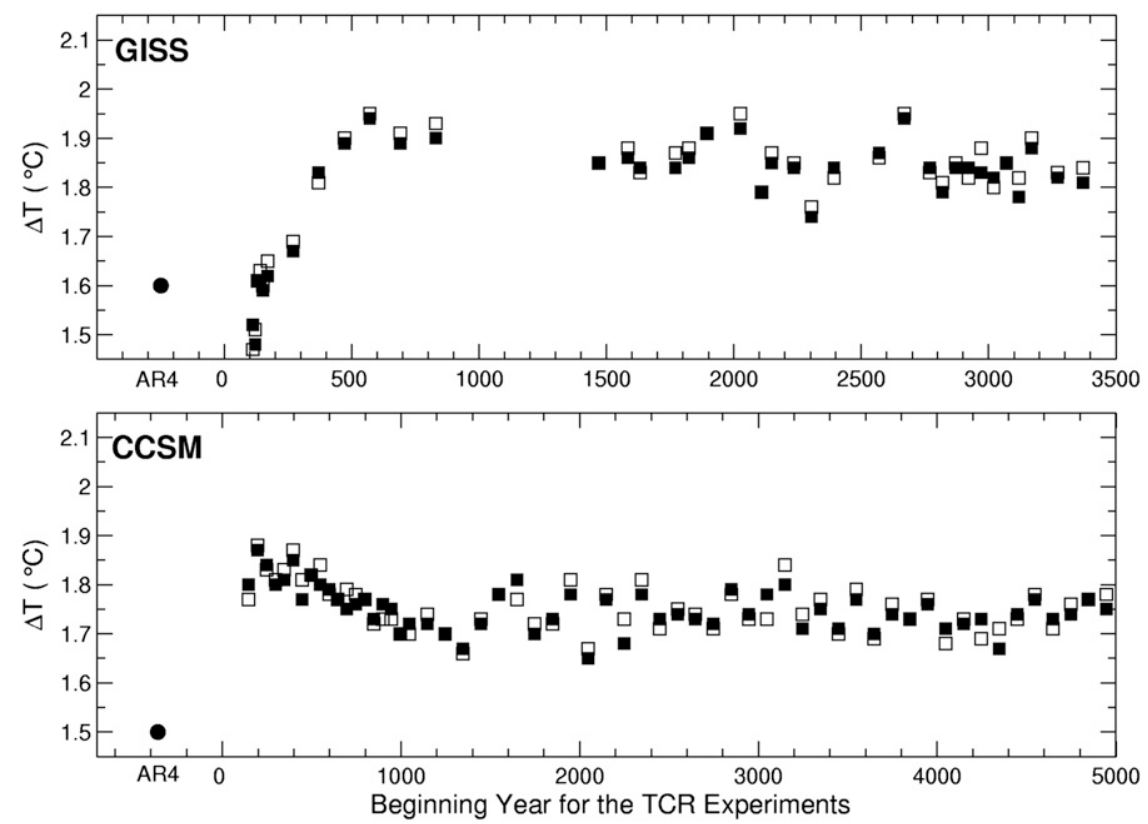

FIG. 4. As in Fig. 2, except with the new definition of TCR: $\Delta T=\bar{T}_{70}-T_{\text {base }}$, where $T_{\text {base }}$ is the 100 -yr average of the control-run temperature prior to the start of each TCR run. 
global change projections are dependent on the inaccurate simulation of the initial mean ocean state, and this might present impediments to projections into the future. Our interpretation is more optimistic, as we argue that this dependence is only a function of protocol.

The remaining TCR variability can be largely removed if we define TCR as $\Delta T=\bar{T}_{70}-T_{\text {base }}$, where $T_{\text {base }}$ is some baseline value for each model; it could be the long-term average of control-run temperature at quasi equilibration. A practical way is to use the 100 -yr mean of the control run prior to (or centered on) year 0. Figure 4 is a recalculation of Fig. 2 using this new definition. We see that the variability is now much reduced: $\Delta T=$ $\bar{T}_{70}-T_{\text {base }}=1.84^{\circ} \pm 0.04^{\circ} \mathrm{C}$ for GISS-EH, and $1.74^{\circ} \pm$ $0.04^{\circ} \mathrm{C}$ for CCSM 4 (spinup period TCRs are not included in the calculation of standard deviation but all are shown in the figure). The $0.1^{\circ} \mathrm{C}$ difference between their TCRs is remarkable given that their ECS difference is 5 times larger (and in the opposite direction) and shows again that the latter is not an important determining factor of the former. Furthermore, the small standard deviations for the prediction of global warming in these forced runs suggest that there is no memory of the initial conditions for these 70-yr runs, since the initial conditions are much more variable (see Fig. 1) than the standard deviation of the final warming. Also, it is not a concern that the long run may lead to different equilibrium states, because these runs branched off initial states that are up to a few thousand years apart and yet the final temperature is about the same.

\section{Conclusions}

We report an underappreciated uncertainty in transient climate simulations: the range of uncertainty in transient climate response introduced by ocean heat uptake variability is about $30 \%$ within the same model, following AR4 protocol.

A large part of the reported intermodel spread of TCR can now be understood as due to various stages of ocean-atmosphere adjustment that the current generation of AOGCMs was in, commonly referred to as "climate drift." The previously adopted remedy of subtracting the control run variability (the drift) is shown here to be ineffective. We recommend that every model control be run at least $1000 \mathrm{yr}$ until quasi equilibrium. Although this recommendation may be viewed as impractical, there does not appear to be an alternative. The variability in TCR can be reduced using our new protocol for their calculation so that it is not so sensitive to the control run unforced variability. This situation is in contrast to the spread of ECS, which probably cannot be reduced further.
Acknowledgments. The research is performed using high performance computing resources provided by ASCC and ASGC. This work is supported in part by NSC Grant 101-2628-M-001-001-MY4 to Academia Sinica. KKT's research is supported by National Science Foundation, under Grant DMS 0940342, and the National Aeronautics and Space Administration, under Grant NNX11AC75G. YLY acknowledges support from the KISS program at the California Institute of Technology.

\section{REFERENCES}

Bitz, C. M., K. M. Shell, P. R. Gent, D. Bailey, G. Danabasoglu, K. C. Armour, M. M. Holland, and J. T. Kiehl, 2012: Climate sensitivity of the Community Climate System Model version 4. J. Climate, 25, 3053-3070.

Bony, S., and Coauthors, 2006: How well do we understand and evaluate climate change feedback processes? J. Climate, 19, 3445-3482.

Brierley, C. M., A. J. Thorpe, and M. Collins, 2009: An example of the dependence of the transient climate response on the temperature of the modelled climate state. Atmos. Sci. Lett., 10, 23-28.

Danabasoglu, G., and P. R. Gent, 2009: Equilibrium climate sensitivity: Is it accurate to use a slab ocean model? J. Climate, 22, 2494-2499.

Gent, P. R., and Coauthors, 2012: The Community Climate System Model version 4. J. Climate, 24, 4973-4991.

Gupta, A. S., L. C. Muir, J. N. Brown, S. J. Phipps, P. J. Durack, D. Monselesan, and S. E. Wijffels, 2012: Climate drift in the CMIP3 models. J. Climate, 25, 4621-4639.

Hansen, J., G. L. Russell, A. Lacis, I. Fung, and D. Rind, 1985: Climate response times: Dependence on climate sensitivity and ocean mixing. Science, 229, 857-859.

Held, I. M., and B. J. Soden, 2000: Water vapor feedback and global warming. Annu. Rev. Energy Environ., 25, 441-475.

Huybers, P., 2010: Compensation between model feedbacks and curtailment of climate sensitivity. J. Climate, 23, 3009-3018.

Meehl, G. A., C. Covey, T. Delworth, M. Latif, B. McAvaney, J. F. B. Mitchell, R. J. Stouffer, and K. E. Taylor, 2007: The WCRP CMIP3 multimodel dataset: A new era in climate change research. Bull. Amer. Meteor. Soc., 88, 1383-1394.

_ , and Coauthors, 2012: Climate system response to external forcings and climate change projections in CCSM4. J. Climate, 25, 3661-3683.

Randall, D. A., and Coauthors, 2007: Cilmate models and their evaluation. Climate Change 2007: The Physical Science Basis, S. Solomon et al., Eds., Cambridge University Press, 589-662.

Roe, G. H., and M. B. Baker, 2007: Why is climate sensitivity so unpredictable? Science, 318, 629-632.

Shindell, D. T., G. Faluvegi, R. L. Miller, G. A. Schmidt, J. E. Hansen, and S. Sun, 2006: Solar and anthropogenic forcing of tropical hydrology. Geophys. Res. Lett., 33, L24706, doi:10.1029/2006GL027468.

Solomon, S., D. Qin, M. Manning, M. Marquis, K. Averyt, M. M. B. Tignor, H. L. Miller Jr., and Z. Chen, Eds., 2007: Climate Change 2007: The Physical Science Basis. Cambridge University Press, 996 pp.

Sun, S., and R. Bleck, 2006: Multi-century simulations with the coupled GISS-HYCOM climate model: Control experiments. Climate Dyn., 26, 407-428. 